\title{
A FORMULA FOR THE HIGH FREQUENCY LONGITUDINAL IMPEDANCE OF A TUBE WITH SMOOTHLY VARYING RADIUS*
}

\author{
Robert L. Warnock \\ Stanford Linear Accelerator Center, Stanford University \\ Stanford, California 94309
}

\begin{abstract}
A formula for the longitudinal coupling impedance at frequencies above or below the tube cut-off is derived. The round tube is infinite in length, and has an arbitrary, smooth variation of radius over a finite interval.
\end{abstract}

\section{DERIVATION OF THE EQUATION}

The method described here may be a useful complement to general purpose programs for computation of fields, especially for high frequency phenomena. Based on a system of integro- algebraic equations, it gives analytic formulas for a first approximation, and also a numerical technique for very accurate solutions. A first report on the analytic and numerical results was given in [1]. Here I review the analytic approach and give an example. The method will be described for the case of the longitudinal impedance in a tube with circular cross section and infinite conductivity. It can be extended to treat transverse fields, wall resistance, and pipes of rectangular cross section.

We take cylindrical coordinates $(r, \phi, z)$ and suppose that the tube radius is given as $R(z)=b-\epsilon s(z)$, where the function $s(z)$ is zero for $|z|>g$, and not necessarily even in $z$. We assume that $s$ has a continuous first derivative $s^{\prime}$, normalized so that $\max \left|s^{\prime}\right|=1$; thus $s^{\prime}( \pm g)=0$. With this normalization, $\epsilon$ measures the effective strength of the wall perturbation; a perturbative method may succeed if $\epsilon$ is small compared to 1 .

We work in the frequency domain, with the time dependence $\exp (-i \omega t)$. Attention is restricted to positive values of $\omega$, which suffice to express the wake field, thanks to the reflection property of the impedance, $Z(\omega)=$ $Z(-\omega)^{*}$. The source is assumed to be axisymmetric, a rigid bunch with total charge $q$ and charge density $\rho(r, \phi, z, t)=(q / 2 \pi) \lambda(z-\beta c t) f(r)$ where $\int \lambda(z) d z=1$, $\int f(r) r d r=1$. It follows that the only non-zero fields are $\left(E_{z}, E_{r}, H_{\phi}\right)$, all independent of $\phi$. All fields may be expressed in terms of $E_{z}$, which can be written as

$$
E_{z}(r, z, \omega)=\int_{\Gamma} d k e^{i k z} a(k, \omega) \frac{I_{o}(\chi r)}{I_{o}\left(\chi^{b}\right)}+e_{z}(r, z, \omega),
$$

where $\chi^{2}=k^{2}-(\omega / c)^{2}$, and $I_{o}$ is the modified Bessel function of the first kind. The Fourier transform $\hat{e}_{z}$ of the source term $e_{z}$ is any particular solution of the inhomogeneous radial wave equation for $E_{z}$, regular at $r=0$. The

* Work supported by the Department of Energy, contract DE-AC03-76SF00515.
Fourier amplitude of the radial field is

$$
E_{r}(r, k, \omega)=-\frac{i k}{\chi^{2}} \frac{\partial E_{z}(r, k, \omega)}{\partial r}
$$

The function $I_{o}\left(\chi^{b}\right)$ has simple zeros in the k-plane at the points $k= \pm k_{s}, s=1,2, \cdots$, where $k_{s}=\left((\omega b / c)^{2}-\right.$ $\left.j_{o s}^{2}\right)^{1 / 2}$ is defined to be positive for $|\omega b / c|>j_{o s}$ and positive imaginary for $|\omega b / c|<j_{o s}$; the $j_{o s}$ are the positive zeros of the Bessel function $J_{o}$. At any frequency above the lowest cutoff $\left(\omega b / c=j_{o 1}\right)$, there are zeros on the real axis. To dodge the corresponding real poles of the integrand in (1), the contour $\Gamma$ follows the real axis but is indented slightly so as to go above the poles at $k=-k$, and below those at $k=k_{s}$. This choice enforces the outgoing wave boundary condition [2]; (see the discussion in the last section of Ref.[1]). We have assumed that $a(k, w)$ is analytic in $k$; our construction of solutions will in fact yield an entire function of $\boldsymbol{k}$.

The boundary condition on the wall is that $\mathbf{E}=$ $\left(E_{r}, E_{z}\right)$ be perpendicular to the tangent vector $(d R, d z)$, or

$$
E_{z}(R(z), z, \omega)+R^{\prime}(z) E_{r}(R(z), z, \omega)=0 .
$$

This condition leads to an equation for $a(k, \omega)$ through the following steps: (i) write $E_{2}$ as in (1), and the corresponding expression for $E_{r}$ constructed from (2); (ii) take the Fourier transform of (3) with respect to $z$; (iii) subtract $I_{o}\left(\chi^{b}\right)$ from $I_{o}(\chi R(z))$ in the integrand, and notice that the compensating addition gives $\delta(k-l)$. The result is

$$
a(l, \omega)=\int_{\Gamma} d k M(l, k, \omega) a\left(k, \omega^{\prime}\right)+\hat{\mathcal{S}}(l, \omega)
$$

where

$$
\begin{aligned}
& M(l, k, \omega)=\frac{1}{2 \pi} \int_{-g}^{g} d z \frac{e^{i(k-l) z}}{I_{o}\left(\chi^{b}\right)} \\
& \cdot\left[I_{o}\left(\chi^{b}\right)-I_{o}(\chi R(z))+\frac{i k}{\chi} R^{\prime}(z) I_{1}(\chi R(z))\right],
\end{aligned}
$$

and $\hat{\mathcal{S}}$ is the Fourier transform of

$$
\begin{aligned}
& \mathcal{S}(z, \omega)=-\int_{-\infty}^{\infty} d k e^{i k z} \\
& \cdot\left[e_{z}(R(z), z, \omega)-\frac{i k}{\chi^{2}} R^{\prime}(z) \frac{\partial e_{z}}{\partial r}(R(z), z, \omega)\right] .
\end{aligned}
$$

Henceforth we treat only the relativistic limit. In that limit the source term takes on the simple form

$$
\mathcal{S}(z, \omega)=\frac{\epsilon Z_{o} s^{\prime}(z)}{2 \pi R(z)} \hat{\lambda}(\omega / c) e^{i \omega z / c} .
$$

DISTPIEUTION OF THIS DOCI IMENT IS UNLIMITED 
Below cutoff, (4) is an integral equation [3] for $a(k, \omega)$. Above cutoff it is an integro-algebraic equation, since the values $a(k, \omega)$ at the poles on the real axis constitute a discrete set of unknowns to be determined along with the continuous, nonpolar part. 'These values determine the amplitudes of outgoing waves.

By reversing the order of integrals we see that any solution of (4) may be written in the form

$$
a(k, \omega)=\frac{1}{2 \pi} \int_{-g}^{g} e^{-i k z} \Phi(z, \omega) d z .
$$

Since the the region of integration is finite, $a(k, \omega)$ is an entire function of $k$, as promised.

\section{FORMULA FOR THE IMPEDANCE}

An integration by parts on the first two terms of (5) puts the kernel in the form

$$
M(l, k, \omega)=\frac{\epsilon}{2 \pi} \frac{k l-(\omega / c)^{2}}{\chi(k-l)} \int_{-g}^{g} d z e^{i(k-l) z} s^{\prime}(z) \frac{I_{1}(\chi R(z))}{I_{o}(\chi b)}
$$

This shows that the kernel is formally $\mathcal{O}(\epsilon)$, and therefore suggests that the equation (4) might be solved by iteraiion when $\epsilon$ is small. The first approximation is obtained by putting $a=\hat{\mathcal{S}}$ under the integral in (4). Since the impedance is proportional to $a(\omega / c, \omega)$, and $\hat{\mathcal{S}}(\omega / c, \omega)=0$, the lowest order impedance is $\mathcal{O}\left(\epsilon^{2}\right)$.

To evaluate the approximated integral of (4) at the synchronous point $k=\omega / c$ we express $\hat{\mathcal{S}}$ in terms of its Fourier transform and reverse integration order to obtain

$$
\begin{aligned}
& a(\omega / c, \omega) \approx \\
& \frac{1}{2 \pi} \int_{-g}^{g} d z e^{-i \omega z / c} R^{\prime}(z) \int_{-g}^{g} d z^{\prime} \mathcal{S}\left(z^{\prime}, \omega\right) K^{\prime}\left(z, z^{\prime}, \omega\right),
\end{aligned}
$$

where

$$
K\left(z, z^{\prime}, \omega\right)=\frac{1}{2 \pi i} \frac{\omega}{c} \int_{\Gamma} d k e^{i k\left(z-z^{\prime}\right)} \frac{I_{1}(\chi R(z))}{\chi I_{o}(\chi b)}
$$

The integral (11) converges exponentially if $R<b$, but diverges for $R>b$. The divergence is an unwanted limitation since we wish to allow arbitrary $R$. By performing a contour distortion one can continue the integral analytically from $R<b$ to $R>b$, and incidentally gain other benefits. Taking $R<b$ we let the contour become an infinite semi-circle in the upper (lower) half-plane for $z-z^{\prime}$ positive (negative). The result is

$$
K\left(z, z^{\prime}, \omega\right)=\frac{\omega}{c} \sum_{s=1}^{\infty} \frac{J_{1}\left(j_{o s} R(z) / b\right)}{k_{s}(\omega) b J_{1}\left(j_{o s}\right)} e^{i k_{s}(\omega)\left|z-z^{\prime}\right|}
$$

For $z \neq z^{\prime}$ the sums converge exponentially, regardless of the value of $R$. At $z=z^{\prime}$ and $R=b$ the sum diverges, but if the integral on $z^{\prime}$ is performed first there will be an extra inverse power of $k_{s}$ and quadratic convergence, uniform in $R$.
The formula (10) now involves powers of $t$ higher than the second through the presence of $R(z)$ in the denominator of (7) and in (12). To pick out just the $\epsilon^{2}$ part we put $R(z)=b$ in both locations. Invoking the usual definition of the impedance in terms of the wake potential, we find $Z(\omega)=-2 \pi a(\omega / c, \omega) /(q \dot{\lambda}(\omega / c))$. Then from (7), (10), and (12) we have the impedance to lowest order in $\epsilon$ as

$$
\begin{aligned}
& Z(\omega)=\frac{\omega Z_{o} \epsilon^{2}}{2 \pi c b^{2}} \sum_{s=1}^{\infty} \frac{1}{k_{s}(\omega)} \int_{-g}^{g} d z \int_{-g}^{g} d u \\
& \cdot s^{\prime}(z) s^{\prime}(u) e^{i k_{\cdot}(\omega)|z-u|-i \omega(z-u) / c} .
\end{aligned}
$$

Below cutoff the $k_{s}$ are all positive imaginary, and the impedance is reactive as required; (the integral is real, since the integrand goes into its complex conjugate on $z \rightarrow u$ ).

A closer look shows that the formula (13) is actually invalid for the frequency $\omega$ in a small neighborhood of each traveling wave cutoff, where $\omega b / c=j_{o s}$. The kernel $K$ has an inverse square-root singularity at such points, owing to the factor $1 / k_{s}$ in (12). It is therefore not small near such frequencies, and the iterative method fails. The same singularity appears in the field expansion (1), from the residue of the pole as it strikes the real axis. It is cancelled by a corresponding zero of $a(k, \omega)$ at $k= \pm k_{s}$, so that the amplitude of the newly appearing outgoing wave is finite. We have verified that this mechanism operates in the numerical calculation of Ref.[1], but it is a "nonperturbative" effect that cannot take place in a lowest order calculation. In plotting results from (13), we delete small neighborhoods of the bad points, and let the plotting program interpolate nearby values to fill in the gaps. This is justified by the smooth beha[ 5 vior of $Z$ found in the numerical solutions.

As an example for arbitrary frequency, we take $s(z)=$ $d(1+\cos (\pi p z / g)) / 2$, where $p$ is an odd integer, thus $\epsilon=$ $\pi p|d| /(2 g)$. An exact evaluation of (13) for this model yields

$$
\begin{aligned}
& Z(\omega)=\frac{\omega b}{c} Z_{o} \frac{\epsilon^{2}}{2 \pi}\left[-i \frac{g}{2 b}[h(\omega)+h(-\omega)]\right. \\
& \left.+\left(\frac{\pi p b}{g}\right)^{2} \sum_{s=1}^{\infty} \frac{1}{b k_{s}(\omega)}\left[f\left(k_{s}(\omega), \omega\right)+f\left(k_{s}(\omega),-\omega\right)\right]\right]
\end{aligned}
$$

where

$$
\begin{gathered}
h(\omega)=\frac{I_{1}(\xi)}{\xi I_{o}(\xi)}, \quad \xi=\left[\left(\frac{\pi p b}{g}\right)^{2}+2 \frac{\omega b}{c}\left(\frac{\pi p b}{g}\right)\right]^{1 / 2} \\
f(k, \omega)=\frac{1-\exp [2 i(k-\omega / c) g]}{b^{4}\left[(k-\omega / c)^{2}-(\pi p / g)^{2}\right]^{2}} .
\end{gathered}
$$

The first term is imaginary at all $\omega$, and so is the sum for $s>s_{m}$. The sum for $s \leq s_{m}$ is complex, and of course present only above cutoff. Its real part is nonnegative as it should be, corresponding to energy lost by the bunch to outgoing waves. The term $h(-\omega)$ has poles, but they are cancelled by corresponding poles in the sum on $s$. 
Figures 1 and 2 show a the real and imaginary parts of formula (14) for $p=5, b=1 \mathrm{~cm}, g=12 \mathrm{~cm}, d=0.12 \mathrm{~cm}$, thus $\epsilon=0.078$. The frequency range (up to $143 \mathrm{GHz}$ ) is sufficient to compute the wake potential of a Gaussian bunch of length $\sigma=1.2 \mathrm{~mm}$. Nothing prevents a treatment of still higher frequencies. Figure 3 shows the wake voltage corresponding to the impedance of Figures 1 and 2 , as a functicn of the distance from the center of the Gaussian bunch (positive in front), in units of $\sigma=1.2 \mathrm{~mm}$; a positive voltage means energy loss. The persistent "ringing" behind the bunch is due to the narrow low frequency resonance $(\omega b / c \approx 2.865)$

In Ref.[1], accuracy of numerical solutions was tested by checking the boundary condition (3). The same can be done in the analytic approach if we compute $a(k, \omega)$ to order $\epsilon^{2}$ for general $k$ then construct the fields from (1).

I wish to thank Robert Gluckstern, Karl Bane, and J. Scott Berg for much good advice and technical help.

\section{REFERENCES}

1. R. L. Warnock, SLAC-PUB 6038 (1993), to be published in AIP Conference Proceedings.

2. A. Sommerfeld, "Partielle Differentialgleichungen der Physik", p.191 (Dieterich'sche Verlagsbuchhandlung, Wiesbaden, 1947).

3. An equation with the same kernel was derived by $S$. S. Kurennoy and S. V. Purtov, Particle Accelerators 36223 (1992). Their form of the source term is incorrect, however, as is their treatment of frequencies above cutoff.
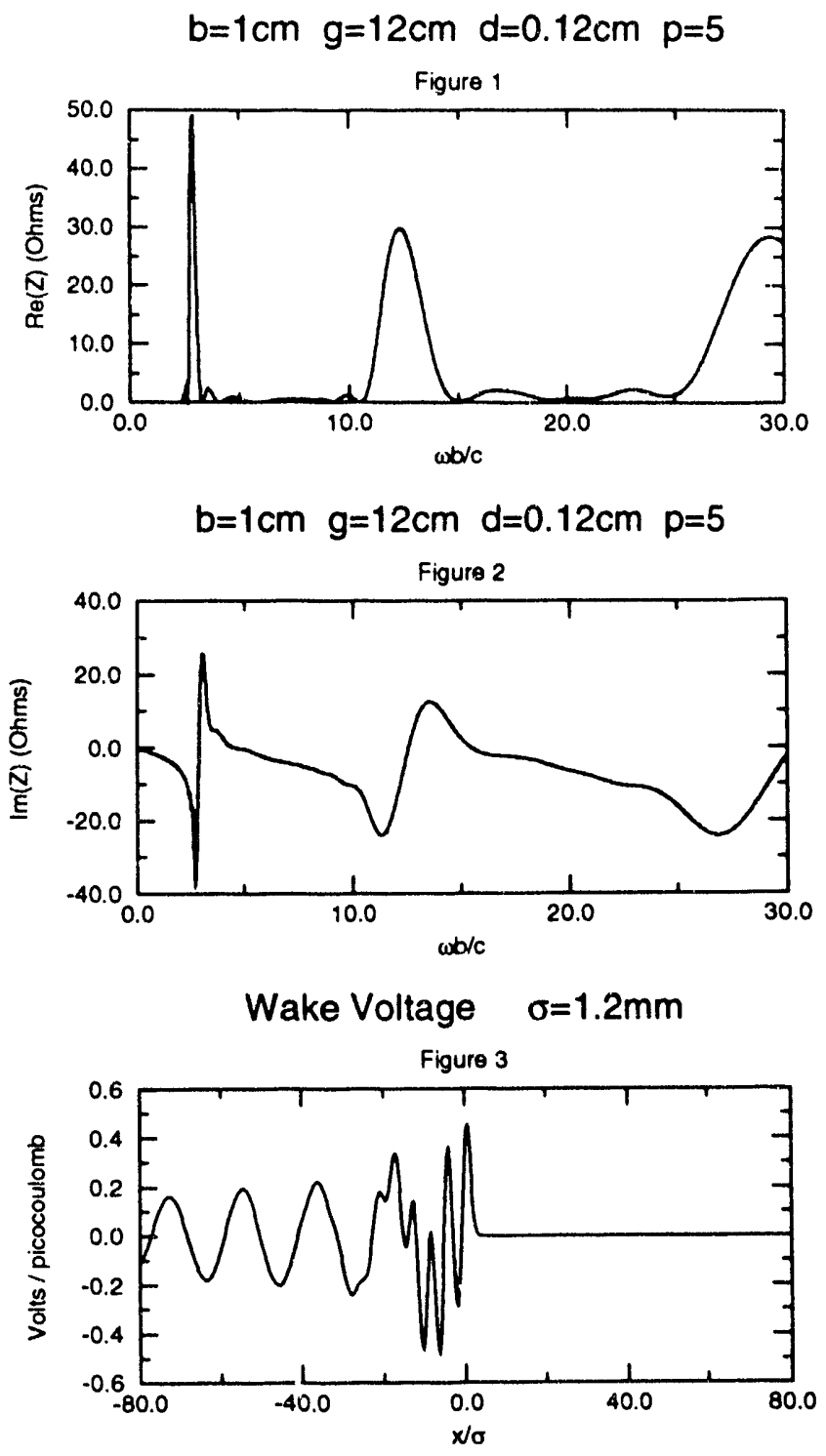

\section{DISCLAIMER}

This report was prepared as an account of work sponsored by an agency of the United States Government. Neither the United States Government nor in ny agency thereof, nor any of their employees, makes any warranty, express or implied, or assumes any legal liability or responsibility for the accuracy, completeness, or usefulness of any information, apparatus, product, or process disclosed, or represents that its use would not infringe privately owned rights. Reference herein to any specific commercial product, process, or service by trade name, trademark, manufacturer, or otherwise does nit necessarily constitute or imply its endorsement, recommendation, or favoring by the "'isited States Government or any agency thereof. The views and opinions of authors expressed herein do not necessarily state or reflect those of the United States Government or any agency thereof. 

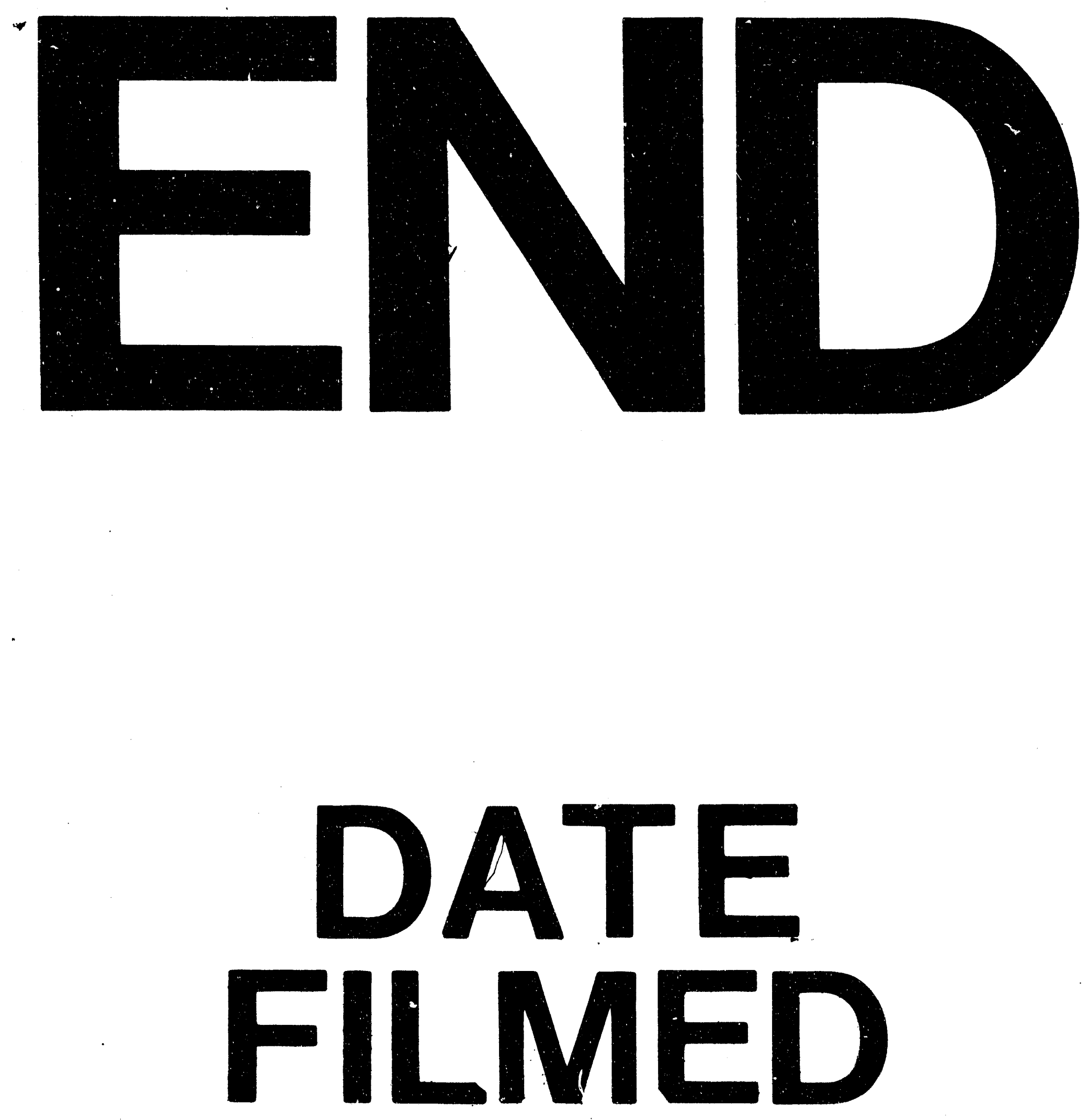

1

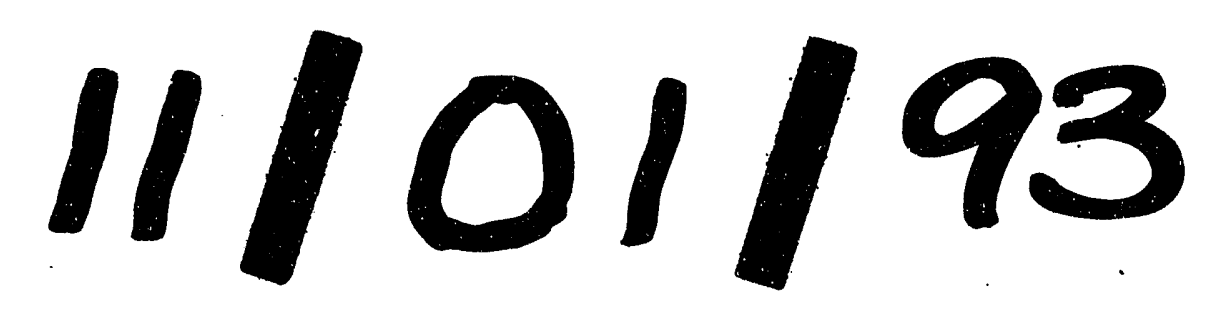


